UCRL 97493

PREPRINT

UCRL --97493

DE88 003293

\title{
Numerical Simulations of Multiphoton Processes \\ in Many Electron Atoms
}

\author{
Kenneth C. Kulander \\ Charles Cerjan
}

\author{
This paper was prepared for submittal to: \\ Proceedings of the NATO Advanced Research Workshop \\ Atomic and Molecular Processes with Short Intense Laser Pulses \\ Bishop's University, Lennoxville, \\ Quebec, Canada \\ July 20-24, 1987
}

September 1, 1987

\section{DISCLAIMER}

This report was prepared as an account of work sponsored by an agency of the United States Government. Neither the United States Government nor any agency thereol, nor any of their employees, makes any warranty, express or implied, or assumes any legal liability or responsibility for the accuracy, completeness, of usefulness of uny information, apparatus, product, or process disclosed, or represents that its use would not infringe privately owned rights. Refer. ence herein to any spectlic commercial product, process, or service by trade nume, Irademark. manufacturer, of otherwise does not necessarily constitute or imply its endorsement, recommendation, or favoring by the United Stules Government or any agency thereol. The views and upinions of authors expressed herein do not necessarily state of renect those of the United States Government or any agency thereof. 
NUMERICAL SIMULATIONS OF MULTIPHOTON PROCESSES IN MANY ELECTRON ATONS

\author{
Kenneth C. Kulander and Charles Cerjan \\ Theoretical Atomic and Molecular Physics Group \\ Lawrence Livermore National Laboratory \\ Livermore, California $\$ 4550$
}

INTRODUCTION

The efferts of high intensity, short pulsed lasers on many electron atoms are actively being studied in a number of laboratories. Measurements of ion yields and electron energy and angular distributions as functions of wavelength, intensity and pulse length have provided detailed information about the process of multiphoton ionization. The pulse lengths and intensities currently used are in the regime where standard perturbative methods are invalid. However, direct solution of the time dependent Schrödinger equation for single electron atoms has been shown to be possible. In this way, arbitrary pulse shapes can be treated exactly as long as the intensity is high enough that ionization occurs rapidly so that long integration times are not required. In this regime, the highly non-linear process encountered can be accurately described. Extensions of these method are now under development, with applications to the simplest multi-electron atom, helium, having been accomplished. $^{2}$ In this report, we will present some details of two numerical methods which we have been pursuing for treating the multi-electron case. Both involve a numerical representation of the time dependent Hartree Fock electronic orbitals and a direct time integration of the coupled equations. Using these methods we will be able to determine the dynamics of the energy absorption process (preionization dynamics and the mechanism of ionization, i.e. whether it is direct multi-electron emission or sequential). 
In the following section, a brief description of the two computational methods will be presented. The final section contains some recent results obtained for the multiphoton ionization of helium along with a discussion of future directions for this research.

\section{NLMERICAL METHODS}

We represent our wave function (or orbital) on a spatial grid and solve a set of coupled equations which gives the values of the wave function at these points at each integration time step. The first method used to accomplish the time propagation will be presented for the single particle, one-dimensional case; the generalization to three dimensions and many electron atoms is discussed below.

We wish to solve the semi-classical time dependent Schrödinger equation for an electron in an electromagnetic field,

$$
i \pi \frac{d}{d t} \psi(x, t)=\left\{-\frac{\pi^{2}}{2 m}\left(\frac{d}{d x}-\frac{e}{c} A(x, t)\right)^{2}+V(x)\right\} \psi(x, t)
$$

where $V(x)$ is the static, field-free potential and $A(x, t)$ is the vector potential. In the dipole approximation, which is valid for all the wavelengths and intensities we have considered, $A(x, t)=A_{0} f(t)$. Since we wish to treat arbitrary pulse shapes, we make no assumptions about the periodicity of the vector potential (as required by Floquet analysis) and solve for the actual time dependent field. We assume the laser field is turned on over several oscillations so that we have $f(t)=f_{0}(t) \operatorname{coswt}$, where $f_{0}$ is a slowly varying function of time, generally chosen to be constant after some turn-on period so that an ionization rate for a particular intensity can be determined.

The method for solving Eq. (1) uses a pseudo-spectral technique. The application of psuedo-spectral methods to the solution of partial differential equations is well-known and extensively developed. 3 The use of a special case of these methods leads to an efficient and accurate technique for the solution of the explicitly time dependent Schrödinger 
equation. 4,5,6 The essence of this technique is to obtain a global representation of the Laplacian differential operator on a grid while remaining in configuration space so that the potential function is just a multiplicative operator. It should be emphasized that the spectral analysis applies only to the spatial derivative operators. The time dependence of the partial differential equation is handled separately--usually by a differencing method. This approach of fers some advantages over standard finite element or finite difference schemes since its extension to many dimensions is automatic and can require fewer grid points than other approaches. The pseudo-spectral method chosen to solve this partial differential equation is the so-called Fast Fourier Transform (FFT) method. The transform is applied to the spatial derivative using the identity:

$$
\frac{d^{n}}{d x} \psi(x, t)=\frac{i^{n}}{2 \pi} \int_{-\infty}^{+\infty} e^{i k x} k^{n} \hat{\psi}(k, t) d k
$$

where the circumflex denotes Fourier transformation for suitably bounded functions $\psi(x)$. Algorithmically, the technique is to transform the wave function on a gird at a given time, multiply by a suitable power of $k$ determined by the order of the spatial derivative and then synthesizing to obtain the spatial derivative on the original grid. Obviously, this method requires two Fourier transforms for every time step. As mentioned above, the time evolution is handled by forward differencing, ${ }^{4}$ or by exponentiation $^{5}$ (split-operator technique). The solution of Eq. (1) in the dipole approximation involves only a first and second order spatial derivative of the wave function.

This method can be generalized easily to higher dimensions and to some other co-ordinate systems. For example, cylindrical or spherical symmetry produces Hankel transforms with the obvious reduction in dimensionality of the problem. The difficulties associated with the method derive from the small time steps needed for numerical stability and similarly for the number of grid points required. The generality of the technique, though, makes this approach a viable and attractive alternative to standard differencing schemes. 
The second approach uses a finite difference representation of the kinetic energy operator. In describing this method, we develop the equations for the time dependent Hartree Fock (TDHF) model of helium in a classical electromagnetic field. In this case we use the E.r rather than the $\rho \cdot A$ interaction shown in Eq. (1). We assume the laser is Linearly polarized in the z-direction so that the Hamiltonian has cylindrical symetry. The two electron, Hartree Fock wave function is given by:

$$
\Psi(\underline{r}, t)=2^{-1 / 2} \varphi\left(\underline{I}_{1}, t\right) \varphi\left(\underline{I}_{2}, t\right)\left\{\alpha_{1} \beta_{2}-\beta_{1} \alpha_{2}\right\}
$$

in the usual notation. Note that in the TDHF model, both electrons occupy the same spatial orbital at all times. This is the major approximation in this treatment, the consequences of wich have been discussed elsewhere. ${ }^{1}$ Putting Eq. (3) into the time dependent Schrödinger equation, we obtain

$$
\text { iÆ } \begin{aligned}
\frac{\partial}{\partial t} \varphi(\underline{r}, t) & =\left\{\left(\pi^{2} / 2 m\right) \nabla^{2}-Z e^{2} / r+e^{2} \int d \underline{r}^{\prime} \rho\left(\underline{r}^{\prime}, t\right) /\left|\underline{r}-r^{\prime}\right|\right. \\
& +e E(t) z \sin w t \mid \varphi(r, t)
\end{aligned}
$$

where

$$
p(r, t)=|\varphi(\underline{r}, t)|^{2}
$$

Taking advantage of the cylindrical symmetry and that the initial state, the static Hartree Fock ground state is $1 \mathrm{~s}^{2}$, we can write

$$
\varphi(\underline{r}, t)=(2 \pi \rho)^{-1 / 2} g(\rho, z, t) .
$$

We define a two dimensional grid in the oz-space and evaluate the second derivatives in the Hamiltonian using a three point formula to 
obtain the following coupled, time-dependent equations for the value of the orbital on the grid points:

$$
\text { if } \frac{\partial}{\partial t} g_{j k}=(H g)_{j k}+\left(V_{g}\right)_{j k}
$$

with

$$
\begin{aligned}
& (H g)_{j k}=-\left(1 / 2 \Delta^{2}\right)\left(g_{j k+1}+g_{j k-1}-2 g_{j k}\right)+\frac{1}{2} w_{j k} g_{j k} \\
& (V g)_{j k}=-\left(1 / 2 \Delta^{2}\right)\left(c_{j} g_{j+1 k}+c_{j-1} g_{j-1 k}-2 g_{j k}\right)+\frac{1}{2} w_{j k} g_{j k}
\end{aligned}
$$

Here $\Delta$ is the grid spacing assumed to be the same in both dinensions,

$$
c_{j}=j /\left(j^{2}-1 / 4\right)^{1 / 2}
$$

and $W$ is the total, laser plus atomic, potential. The time propagation is accomplished using the Peaceman-Rachford (PR) alternating directions implicit method given by:

$$
g^{(n+1)}=[I+i \tau V]^{-1}[I-i \tau H][I+i \tau H]^{-1}[I-i \tau V] g^{(n)}
$$

where $I$ is the unit matrix, $g^{(n)}$ is the orbital at $t=n \Delta t$, and $\tau=\Delta \tau / 2 h$. Since the Fock Hamiltonian depends on the time through the Coulomb term

$$
v_{c}(\underline{r}, t)=\int d \underline{r}^{\prime} \rho\left(\underline{I}^{\prime}, t\right) /\left|\underline{r}-\underline{r}^{\prime}\right|
$$


and this depends in turn on the orbital, we use a two step, predictor-corrector type integration. First we propagate to $t_{n+1}$ to obtain $\bar{w}^{(n+1)}$ which is the interaction evaluated using the extrapolated $g^{(n+1)}$, then use

$$
W^{(n+1 / 2)}=\frac{1}{2}\left[W^{(n)}+W^{(n+1)}\right]
$$

to propagate the next time step. We have found this to give accurate wave function evolution with reasonable time steps. The propagator defined in Eq.(10) is unitary, second order in time and involves either the multiplication by or the inversion of tri-diagonal matrices. These are accomplished by a few vector multiplications so that the calculations are very efficierit.

The evaluation of the Coulomb integral is a major part of the calculation. From Eq. (11) it can be seen that it must be determined for each grid point for each time integration step. We do this by first performing a moment expansion of the density using Legendre polynomials which is then used to explicitly evaluate $v_{c}$ for the points on the grid boundary. Values for the balance of the grid points are determined by solving the differential equation

$$
\nabla^{2} v_{c}(\underline{r}, t)=-4 \pi \rho(\underline{r}, t)
$$

using an iterative procedure similar to the PR algorithm.

Thus, the calculation consists of choosing a laser frequency, a pulse shape and a maximum intensity; then solving for the time propagation using Eq. (10) subject to the initial condition of the atom being in its ground state. The wave function evolution is followed until an ionization rate can be determined in cases where our exponential decay of the initial state occurs. Since the final state generally corresponds to free electrons, some accommodation for the finite size of the numerical grid must be made. We do this by including an imaginary part to the potential defined only on a band around the edge of the grid which 
absorbs all flux which reaches the boundary. Therefore all probability which is excited enough to reach the boundary is assumed to be ionized. Then the ionization rate is computed by determining the change in the norm of the total wave function due to the absorption of this flux. We check the sensitivity of the results to the size of the grid, i.e. the distance from the nucleus to the boundary, to assure the box is large enough.

We have performed a number of calculations on the helium system for various wavelengths and intensities. A sample of the results are presented in the next section.

\section{RESULTS AND CONCLUSIONS}

We have determined the multiphoton ionization rates for helium at wavelengths commonly employed in these experiments--1064, 532 and $248 \mathrm{~nm}$. Intensities in the range from $10^{14}-2 \times 10^{15} \mathrm{~W} / \mathrm{cm}^{2}$ were used. We chose a pulse shape [see Eq. (4)] defined by $E(t)=E_{\max } f(t)$, where

$$
f(t)= \begin{cases}1 / t_{\max }, & t<t_{\max } \\ 1, & t>t_{\max }\end{cases}
$$

$t_{\text {max }}$ is chosen to be a small number of periods of the laser, but large enough that the turn-on for these wavelengths is adiabatic. In these cases we found an exponential decay of the wave function during the constant intensity interval.

The calculated rates are shown in the accompanying table. We note two interesting facts about these results. First, the rates are comparable in magnitude although the order of the ionization process (i.e. the minumum number of photons required to create an ion) change significantly from 22 to 5 . As the intensity increases, we find the rates become independent of wavelength. Second, the rates do not increase as $I^{n}$ where $n$ is the order of the process as would be predicted by perturbation theory. Clearly, these results are in the regime where perturbation theory is invalid. This should be expected since the electric field due to the laser is becoming comparable to the Coulomb interactions in the atom so that the electromagnetic field cannot be considered a weak perturbation. 
These results illustrate the computational techniques described above. More extensive results have been presented and discussed elsewhere. ${ }^{2,4}$ The methods are being generalized to treat the multiphoton ionization of $\mathrm{Xe}$. In this case, we explicitly solve for the outer valence shell $\left(5 s^{2} 5 p^{6}\right)$ electrons with the core represented by a local pseudopotential. Exchange terms are present in this case and are included also by a local density approximation. In this way we can examine the multi-electron character of the pre-ionization dynamics.

A second direction of development is the inclusion of a second configuration in the helium calculation so that the electrons can interact with the field independently. The restriction of a single determinantal wave function means we cannot investigate the role of direct double Ionization relative to sequential ionization for producing completely stripped helium.

We conclude by emphasizing that the methods described here show great promise for detailed investigation of the dynamics of many-electron atoms in intense laser fields.

Table 1. Multiphoton ionization rates $\left(\mathrm{s}^{-1}\right)$ for helium as functions of wavelength $(\mathrm{nm})$ and intensity $\left(W / \mathrm{cm}^{2}\right)$

\begin{tabular}{cccc}
\hline$I \backslash \lambda$ & 1064 & 532 & 248 \\
\hline $5 \times 10^{14}$ & $4.7 \times 10^{11}$ & $9.0 \times 10^{11}$ & $3.9 \times 10^{12}$ \\
$1 \times 10^{15}$ & $1.5 \times 10^{13}$ & $1.9 \times 10^{13}$ & $2.5 \times 10^{13}$ \\
$2 \times 10^{15}$ & $1.2 \times 10^{14}$ & $1.4 \times 10^{14}$ & $1.8 \times 10^{14}$ \\
\hline
\end{tabular}

\section{ACKNOWLEDGEMENT}

Work performed under the auspices of the U. S. Department of Energy by the Lawrence Livermore National Laboratory under contract number W.7405-ENG-48. 


\section{RFFERENCES}

1. K. C. Kulander, Multiphoton Ionization of Hydrogen: A Time Dependent Theory," Phys. Rev. A 35445 (1987).

2. K. C. Kulander, "Time Dependent Hartree Fock Theory of Multiphoton Ionization: Helium," Phys. Rev. A 362726 (1987).

3. D. Gottlieb, M. Y. Hussaini and S. A. Orszag, "Theory and Applications of Spectral Methods" in Spectral Methods for Partial D. Differential Equations, ed. by R. G. Voigt, D. Gottlieb and M. Y. Hussaini, SIAM publications (Philadelphia, $1984)$.

4. C. Cerjan and R. Kosloff, "Non-Perturbative Treatment of Particle Dynamics in a Semi-Classical Photon Field," J. Phys. B 204441 (1987).

5. M. D. Feit, J. A. Fleck and A. Steiger, "Solution of the Schrödinger Equation by a Spectral Method," J. Comp. Phys. 47 412 (1982).

6. H. Tal-Ezer and R. Kosloff, "An Accurate and Efficient Scheme for Propagating the Time Dependent Schrodinger Equation," J. Chem. Phys. 813967 (1984).

br/0201K 